\title{
Polinización efectiva de flores ornitófilas en un bosque de niebla de Colombia
}

\author{
Effective pollination of ornitophylous \\ flowers in a cloud forest of Colombia
}

Angie Lisseth Martinez-Meneses ${ }^{\mathrm{a}}$

Alba Marina Torres-González

Fecha de Recepción: 17.12.2019

Fecha de Aceptación: 03.03.2020

Doi: https://doi.org/10.19053/01217488.v11.n2.2020.10466

\section{Resumen}

La interacción planta-colibrí es fundamental para la polinización en los bosques de niebla. Esta investigación evaluó la efectividad de la polinización por colibríes en cuatro especies de plantas en la Finca Zíngara, Cali, Colombia. Se evaluaron las visitas de colibríes, la reproducción de las plantas, la oferta energética de las flores y la deposición de polen en los estigmas. Se encontró que Guzmania multiflora fue la especie más visitada por colibríes, seguida por Cavendishia adenophora. En Columnea ferruginea y Columnea anisophylla sólo se observaron cuatro y una visita de una especie de colibrí ermitaño, respectivamente. Las cuatro especies de plantas presentan alogamia, Guzmania multiflora y Cavendishia adenophora son autocompatibles, mientras que las dos especies de Columnea son autoincompatibles. El polen que depositan los colibríes en el estigma es principalmente legítimo (Guzmania multiflora: 77,8\%, Cavendishia adenophora: $51,6 \%$ ). El mayor volumen de néctar acumulado en el día fue producido por Guzmania multiflora: 28,53 $\mu 1$ y Cavendishia adenophora: 23,91 $\mu$. La mayor concentración de néctar la produjeron Columnea ferruginea: 20,14\% y Cavendishia adenophora: 17,4\%. Se concluye que los colibríes son polinizadores efectivos de Guzmania multiflora y Cavendishia adenophora. Además, para los colibríes en este estudio fue más importante visitar flores de las especies que producen mayor cantidad de néctar, que flores de especies con mayor concentración de sacarosa.

Palabras clave: epifluorescencia, mutualismo, néctar, polen, tubo polínico.

\begin{abstract}
Bióloga con énfasis en Botánica. Departamento de Biología. Universidad del Valle. Cali, Colombia.

*_angie.1.martinez@correounivalle.edu.co

b Bióloga, M.Sc., Ph.D. Departamento de Biología. Universidad del Valle. Cali, Colombia.

* alba.torres@correounivalle.edu.co
\end{abstract}

The plant-hummingbird interaction is fundamental for pollination in cloud forests. This research evaluated the effectiveness of pollination by hummingbirds in four species of plants in the Finca Zingara, Cali, Colombia. We assessed the hummingbird visits, the reproduction of the plants, the flowers' energetic offer, and the deposition of pollen on the stigmas. We found that Guzmania multiflora was the most visited species by hummingbirds, followed by Cavendishia adenophora. In Columnea ferruginea and Columnea anisophylla we observed only four and one hermit hummingbird visits, respectively. The four species exhibit allogamy, Guzmania multiflora, and Cavendishia adenophora are auto compatible. Meanwhile, the two Columnea species are auto incompatible. The pollen that hummingbirds lay on the stigma is mainly legitime (Guzmania multiflora: 77,8\% and Cavendishia adenophora: 51,6\%). The higher volume of nectar accumulated during the day was produced by Guzmania multiflora: 28,53 $\mu 1$ and Cavendishia adenophora: 
23,91 $\mu$ l. The higher sucrose concentration in nectar was produced by Columnea ferruginea: $20,14 \%$ and Cavendishia adenophora: :17,4\%. We conclude that hummingbirds are the effective pollinators of Guzmania multiflora and Cavendishia adenophora. Furthermore, for the hummingbirds in this study, it was more important to visit flowers of the species that produce the highest amount of nectar, than flowers of species with the highest sucrose concentration.

Keywords: epifluorescence, mutualism, nectar, pollen, pollen tube.

\section{INTRODUCCIÓN}

Las flores ornitófilas presentan morfologías diversas que están relacionadas con la morfología de los picos de los colibríes [1]. Las familias de plantas Rubiaceae, Gesneriaceae, Campanulaceae, Ericaceae y Bromeliaceae, se identifican por características que se conocen como síndrome de ornitofilia: antesis diurna, corola tubular, color rojo, sin olor, ausencia de guías nectaríferas y néctar abundante [2]. Sin embargo, los colibríes pueden explotar flores que no cumplen con este síndrome, ya que pueden ser oportunistas en el uso del recurso [3].

Los colibríes (Aves: Trochilidae) son los vertebrados con el metabolismo más alto y para mantenerlo se alimentan principalmente de néctar de flores, que es un elemento con alto contenido calórico y de fácil digestión [4].

El néctar está compuesto principalmente por una solución de tres tipos de azúcares: sacarosa, fructosa y glucosa, en cantidades variables. En el caso de los colibríes, ellos prefieren flores con mayor concentración de sacarosa [5]. Las medidas de volumen, concentración del néctar y la distribución espacial de las flores permiten entender los requerimientos energéticos, el comportamiento del polinizador y su movimiento en parches florales que, a su vez, afectan la dispersión del polen y la reproducción de las poblaciones de plantas [6].

El primer paso para que ocurra la reproducción sexual en las plantas angiospermas es la polinización, que en sentido estricto, es la transferencia de polen de las anteras al estigma. La función principal del polen es la fecundación de los óvulos, que emerge por crecimiento del ápice del tubo polínico [7]. El crecimiento del tubo ocurre a una velocidad de varios milímetros por hora, dependiendo tanto de la especie como de las condiciones climáticas y de la calidad del polen [8].

Por otro lado, los sistemas de reproducción de las plantas autofecundación, fecundación cruzada y la fecundación mixta, están determinados por la presencia o ausencia de órganos femeninos y masculinos y el grado de autoincompatibilidad [9]. Por lo tanto, el éxito reproductivo de las plantas se relaciona con la eficiencia tanto de la polinización como de la fecundación del óvulo.

La interacción planta-colibrí constituye un componente importante en los bosques de niebla [1]. En la actualidad, los bosques de niebla son una de las principales prioridades de conservación mundial, siendo reconocidos como uno de los hotspots de biodiversidad global [10]. A pesar de ello, estos bosques están es un estado frágil de gran presión y amenaza global [11]. Aún es poco entendido cómo la amenaza a los bosques afectaría los procesos ecológicos tales como la polinización (e.g. plantas polinizadas por colibríes), la cual es esencial para el funcionamiento y el mantenimiento a largo plazo de los ecosistemas naturales y de la diversidad biológica [12].

La eficiencia de la polinización ha sido poco estudiada, ya que se ha dado un mayor enfoque a la interacción planta-colibrí desde la parte zoológica, en procesos ecológicos como la polinización [13, 14] y evolutivos como la correspondencia picocorola $[15,16,17]$.

Además, en Colombia, se han realizado estudios desde una perspectiva palinológica, tanto en localidades de la región alto andina [18] donde son frecuentes las áreas fragmentadas, como en áreas de bosque húmedo tropical en la Amazonía [19, 20, 21].

Por otro lado, la efectividad de la polinización se ha evaluado principalmente en zonas de selvas tropicales [22, 23, 24] y en el Cerrado de Brasil [25]. En este sentido, es necesario conocer cómo es la interacción planta-colibrí, en aspectos como la carga de polen legítimo del colibrí que es depositada en el estigma de la flor en un bosque de niebla, lo cual es un indicador de la efectividad de la polinización. En consecuencia, esta investigación tuvo como objetivo evaluar el sistema reproductivo y la efectividad de la polinización por colibríes en cuatro especies de plantas en un bosque de niebla en la cordillera occidental de los Andes de Colombia. 


\section{MATERIALES Y MÉTODOS}

\section{1 Área de estudio}

Este estudio se realizó en la finca Zíngara (332'N, 76³6'W), a 1.900-2.000 m de altitud, en la zona rural del corregimiento La Elvira, al noroccidente de la ciudad de Cali, Valle del Cauca, en la vertiente oriental de la cordillera occidental de los Andes de Colombia. La parte alta de la finca presenta un bosque en buen estado de conservación, y tiene un jardín con plantas nativas del bosque y plantas ornamentales introducidas. La zona de vida corresponde a bosque montano bajo, según la clasificación de Holdridge. La temperatura media en esta zona es $16^{\circ} \mathrm{C}$ y fluctúa entre los 12 y $20{ }^{\circ} \mathrm{C}$.

Para realizar las observaciones, en el bosque se delimitaron cinco transectos de $20 \mathrm{~m}$ x $1 \mathrm{~m}$ (i.e. $100 \mathrm{~m}^{2}$ ), y en la zona de jardín se delimitaron 10 transectos de $10 \mathrm{~m}$ x $1 \mathrm{~m}$ (i.e. $100 \mathrm{~m}^{2}$ ), para un total de $200 \mathrm{~m}^{2}$.

\subsection{Visitas de colibríes a las plantas}

Se eligieron cuatro especies de plantas nativas del bosque de niebla: Guzmania multiflora (André) André ex Mez (Bromeliaceae), Cavendishia adenophora Mansf. (Ericaceae), Columnea ferruginea J.F. Sm. \& J.L. Clark (Gesneriaceae) y Columnea anisophylla DC. (Gesneriaceae), (Figura 1). Se verificó que las flores de estas especies fueran abundantes y que fueran visitadas por colibríes. Las especies fueron identificadas por comparación con especímenes botánicos en el Herbario CUVC, de la Universidad del Valle.

Se observaron las visitas de colibríes a las flores de las cuatro especies de plantas seleccionadas, entre marzo y diciembre de 2016, entre 7:00 y 17:00 h. con el uso de binoculares Nikon 8 x 42 . La identificación de los colibríes se realizó con Ayerbe [26].

Durante las observaciones se registraron las especies de colibríes que visitaron las flores, incluyendo el número de flores probadas y la hora de la visita. Las observaciones en Cavendishia adenophora se realizaron durante 54 horas, en 5 individuos con 322 flores en antesis; en Guzmania multiflora durante 72 horas, en 21 individuos con 343 flores en antesis; en Columnea ferruginea durante 32 horas, en 2 individuos con 5 flores en antesis; y en Columnea anisophylla durante 16 horas, en 4 individuos con 65 flores en antesis. En total se realizaron 174 horas de observación a las visitas de colibríes, en 32 individuos con 735 flores en antesis, para las cuatro especies de plantas seleccionadas.

\subsection{Sistema reproductivo de las especies de plantas}

Se cubrieron botones florales con una bolsa de muselina 24 horas previas a la antesis para realizar los tratamientos: 1) polinización cruzada natural (control de alogamia): las flores se marcaron y se dejaron expuestas a los polinizadores. 2) Polinización cruzada obligada: se emascularon los botones florales, se aislaron las flores de los polinizadores con bolsas de muselina y se polinizaron manualmente con polen de flores de otra planta. 3) Autopolinización natural (control de autogamia): las flores se aislaron de los polinizadores con bolsas de muselina y no se hizo ningún tipo de manipulación. 4) Autopolinización obligada: se emascularon los botones florales, se aislaron de los polinizadores con bolsas de muselina y las flores se polinizaron manualmente con polen producido por flores de la misma planta. Para evaluar el éxito de estos tratamientos, se usaron 40 y 20 flores de Cavendishia adenophora, 20 y 20 de Guzmania multiflora, 8 y 8 de Columnea ferruginea, y, 8 y 5 de Columnea anisophylla, para determinar el porcentaje de formación de frutos y el crecimiento de tubos polínicos, respectivamente.

Después de 24 horas de iniciado cada tratamiento, se colectaron las flores, se sumergieron durante una hora en solución AFA (Alcohol 50\%: 18; Formol 10\%: 1; Ácido acético glacial 10\%:1) [6], y se conservaron en recipientes plásticos con $100 \mathrm{ml}$ de alcohol al 70\% y 30 gotas de glicerina. En el laboratorio de Semillas de la Universidad del Valle, las flores se cortaron longitudinalmente con un bisturí, se extrajeron los pistilos, se depositaron en cajas de Petri plásticas y se cubrieron con hidróxido de sodio ( $\mathrm{NaOH}: 8 \mathrm{~N}$ ) para aclararlos [27]. El tiempo de aclaración fue de 24 horas para Guzmania multiflora, 48 para Columnea anisophylla, 72 para Cavendishia adenophora y 72 para Columnea ferruginea. Posteriormente, los pistilos se lavaron cuidadosamente con agua destilada para remover el hidróxido de sodio.

Se usó el colorante azul de anilina al 0,01\% en $0,1 \mathrm{~N}$ de $\mathrm{K}_{3} \mathrm{PO}_{4}$ para colorear los pistilos de acuerdo con el protocolo establecido por Shivanna [27]. 
La tinción se realizó en cajas de Petri de 60 x 15 $\mathrm{mm}$, cubiertas con papel aluminio para evitar el contacto con la luz. Los pistilos se trasladaron a las cajas Petri con un palillo de madera, se cubrieron con la solución de azul de anilina, se taparon, y se registró la fecha y hora para cada tratamiento. Esta tinción se dejó durante 48 horas de acuerdo con Murillo [28].

Los pistilos se colocaron sobre un portaobjetos y se cubrieron haciendo una presión suave con el cubreobjetos. Los portaobjetos fueron rotulados, cubiertos con papel aluminio para evitar el contacto con la luz, guardados en bolsa plástica negra, y almacenados para su conservación temporal en nevera a $5{ }^{\circ} \mathrm{C}$.

En el laboratorio de imágenes de la Universidad del Valle, los pistilos teñidos se observaron en un microscopio Nikon triocular, con epifluorescencia intensilight, modelo Eclipse Ni-U 90, para comprobar el crecimiento de tubos polínicos. Se tomaron fotos del estigma, estilo y ovario, con una cámara digital Nikon, modelo DS-Ri1 U3 integrada al microscopio.

\subsection{Reconocimiento de polen legítimo e ilegítimo depositado por los colibríes en los estigmas}

Para facilitar la identificación específica del polen, se hizo una colección de referencia de polen de todas las plantas ornitófilas en floración. Con una pinza se colectó polen de las anteras de flores previamente aisladas con bolsas de muselina, se depositó en un portaobjetos con una gota de gelatina glicerinada teñida con fucsina y se cubrió con un portaobjetos [29]. Las muestras de polen se calentaron con un encendedor por la parte inferior del portaobjetos para derretir la gelatina, sin dejarla hervir. Luego, se aplicó alrededor del cubreobjetos una capa de barniz transparente para fijarla temporalmente y se rotuló cada muestra de polen semipermanente.

Se emascularon 35 botones florales de Guzmania multiflora y Cavendishia adenophora y 6 botones florales de Columnea ferruginea y Columnea anisophylla, se cubrieron con bolsas de muselina, y cuando ocurrió la antesis se quitó la bolsa. Las flores emasculadas se observaron hasta el momento en que un colibrí visitó cada flor. Inmediatamente después, con un palillo de madera se retiró el polen que el colibrí dejó en el estigma. El polen se depositó sobre un portaobjetos con una gota de gelatina glicerinada teñida con fucsina y se cubrió con un cubreobjetos [29]. Luego, se hizo el mismo procedimiento para hacer muestras de polen semipermanentes, explicado arriba. Finalmente, se identificaron las muestras de polen depositadas por los colibríes en el estigma de las flores de las cuatro especies de estudio [6]. Se hizo identificación del polen por comparación con la colección de referencia, lo que permitió definir el polen legítimo (i.e. polen de la misma especie) y el polen ilegítimo (i.e. polen de otra especie). Se determinó la presencia/ausencia de polen legítimo/ ilegítimo con un microscopio óptico OLYMPUS, modelo CX21 FS1.

\subsection{Producción del néctar acumulado en las flores}

En 10 a 15 botones florales de cada una de las especies estudiadas se cubrieron con bolsas de muselina un día antes de la antesis. El día de la antesis se midió el volumen del néctar acumulado durante todo el día (i.e. 16:00 horas). La extracción y medición del néctar se hizo con tubos microcapilares aforados en microlitros de $50 \mu 1$ de capacidad total. Se midió la concentración del néctar extraído en grados Brix, que corresponde al porcentaje de sacarosa, con un refractómetro de mano VEE GEE, modelo BTX-1 (Brix 0-32\%).

\section{RESULTADOS Y DISCUSIÓN}

\subsection{Visitas de colibríes a las plantas}

Se registraron nueve especies de colibríes que visitaron las cuatro especies de plantas durante el período de estudio (Figura 2). Se observó variación en el número de flores que fueron visitadas por colibríes. La especie Guzmania multiflora fue la más visitada, con 415 visitas de cinco especies de colibríes. El registro más alto de visitas a flores de Guzmania multiflora fue del colibrí Adelomyia melanogenys (72,5\%), seguido de Calliphlox mitchellii $(18,8 \%)$ (Figura 2). Estos dos colibríes mostraron un comportamiento territorial durante todas las visitas a esta especie. Según Murcia [14] una característica de los sistemas de polinización en Bromeliaceae es la redundancia, lo que significa que varias especies de polinizadores visitan la misma especie de planta o varias plantas son 
visitadas por el mismo polinizador, tal como ocurrió en Guzmania multiflora.

La segunda especie de planta más visitada fue Cavendishia adenophora con 352 visitas de seis especies de colibríes. El colibrí que realizó más visitas a Cavendishia adenophora fue Adelomyia melanogenys (36,4\%), seguido de Coeligena coeligena (19,3\%) y Amazilia franciae (17,3\%) (Figura 2).

Mientras que, en Columnea ferruginea solamente se observaron cuatro visitas del colibrí Phaethornis syrmatophorus, y en Columnea anisophylla se registró una visita del colibrí Phaethornis guy (Figura 2). Estas especies de Columnea presentaron flores protándricas con corolas tubulares que limitan las características de los visitantes florales. Esto explica el hecho que los colibríes ermitaños Phaethorninae, fueron los únicos visitantes de Columnea spp., pues con su pico largo y curvo, pudieron alcanzar la fuente de néctar de estas flores. Este resultado coincide con Marin \& Amaya [30], que reportan a las especies del género Columnea, con baja intensidad en la floración y polinizadas por colibríes ermitaños. Sin embargo, Columnea anisophylla presenta características de floración contrastantes, crece hacia los bordes del bosque y tiene un mayor número de flores que Columnea ferruginea, lo cual concuerda con el estudio de Ackerman [31]. El bajo número de visitas de colibríes registradas a las flores de ambas especies de Columnea puede disminuir drásticamente la polinización exitosa de sus flores en esta localidad.

La composición y abundancia de las visitas de colibríes a las flores de cada especie en la finca Zíngara varió en el tiempo. En Guzmania multiflora y Cavendishia adenophora las visitas menos frecuentes la tuvieron los colibríes Calliphlox mitchellii, Haplophaedia aureliae, Ocreatus underwoodii, Coeligena coeligena, Amazilia franciae, Florisuga mellivora, Phaethornis guy y Phaethornis syrmatophorus (Figura 2). Esto muestra la variación en el comportamiento de forrajeo de los colibríes que componen una determinada comunidad [15].

\subsection{Sistema reproductivo de las especies de plantas}

En las especies de plantas Guzmania multiflora y Cavendishia adenophora se obtuvo formación de frutos en todos los tratamientos, a excepción de la autopolinización natural. Sin embargo, debido al éxito en la autopolinización obligada en ambas especies, se consideran autocompatibles (Tabla 1).

La autocompatibilidad de Guzmania multiflora concuerda con Bush \& Beach [32], quienes reportan a todas las especies de Bromeliaceae en los bosques de niebla como autocompatibles. Por otro lado, la autocompatibilidad de Cavendishia adenophora, y su presencia en zonas abiertas en el jardín de la finca Zíngara, coincide con que la mayoría de las especies de Ericaceae son pioneras de ambientes húmedos y fríos, y colonizan fácilmente ambientes en restauración o áreas que han sido perturbadas [33]. Estos resultados también confirman a Bawa [34], quien reporta en plantas pioneras una alta autocompatibilidad.

Por otro lado, en Columnea ferruginea solamente se obtuvo fructificación en el tratamiento de polinización cruzada natural y en Columnea anisophylla se obtuvo fructificación en la polinización cruzada natural y obligada (Tabla 1). La ausencia de fructificación en los dos tratamientos de autopolinización en las dos especies de Columnea muestra que estas dos especies son autoincompatibles. El sistema principalmente autoincompatible en especies de Columnea de sotobosque también fue reportado por Marin \& Amaya [30].

Por otro lado, en las fotografías tomadas en el microscopio de epifluorescencia, se observó crecimiento de tubos polínicos en los tratamientos de autopolinización obligada en Guzmania multiflora (Figura 3A) y polinización cruzada obligada en Guzmania multiflora (Figura 3C), Columnea anisophylla (Figura 3E) y Columnea ferruginea (Figura 3F). Este crecimiento de tubos polínicos desde el estigma hasta el ovario confirma el éxito de formación de frutos para los mismos tratamientos de polinización en estas especies. Además, en el tratamiento autopolinización natural, se observó crecimiento de tubos polínicos solamente hasta el estilo en Guzmania multiflora (Figura 3B), y ausencia de polen en el estigma de Cavendishia adenophora (Figura 3D), que confirman el fracaso en la formación de frutos para este tratamiento en estas especies.

\subsection{Reconocimiento de polen legítimo e ilegítimo depositado por los colibríes en los estigmas}

La frecuencia de polen legítimo, es decir, de la misma especie de planta, depositado en los estig- 
mas de flores de Guzmania multiflora fue 77,8\%, mientras que un $22,2 \%$ fue polen ilegítimo. Para Cavendishia adenophora el 51,6\% de polen registrado fue legítimo y el 48,4\% fue polen ilegítimo. En Columnea ferruginea y Columnea anisophylla, no se logró obtener polen depositado en sus estigmas, debido a la poca disponibilidad de flores para esta prueba. Además, los colibríes ermitaños que visitaron estas dos especies de Columnea, lo hicieron de manera esporádica durante el tiempo de observación.

La transferencia de polen interespecífica puede reducir el éxito reproductivo de las plantas involucradas, debido a que granos de polen heterospecíficos llegan a la superficie del estigma e interfieren en la germinación del polen conespecífico [35]. Esta transferencia de polen intruso afecta de alguna manera el fitness de las plantas [36].

En plantas de Bromeliaceae, hay una secuencialidad en la floración y por ende una alta eficiencia en la polinización [37], especialmente en bromelias ornitófilas $[38,39,40]$. Esta secuencialidad en la floración probablemente minimiza la competencia por polinizadores y aumenta la efectividad en la transferencia de polen [41]. De hecho, en este estudio Guzmania multiflora fue la especie de planta más visitada por colibríes, mostró autogamia y alogamia exitosa y alto valor de polen legítimo $(77,8 \%)$ depositado en el estigma por los colibríes, confirmando la eficacia de la polinización en esta especie.

\subsection{Producción de néctar acumulado en las flores}

El néctar tuvo mayor concentración de sacarosa en las flores de Columnea ferruginea $(\dot{X}=20,14 \%)$ y Cavendishia adenophora ( $\dot{\mathrm{X}}=17,4 \%)$, mientras que tuvo menor concentración en Columnea anisophylla ( $\dot{X}=16,44 \%)$ y Guzmania multiflora ( $\dot{X}=15,74 \%$ ), (Figura 4). El volumen de néctar acumulado en el día fue más abundante en Guzmania multiflora $(\dot{\mathrm{X}}=28,53 \mu \mathrm{l})$, seguido de Cavendishia adenophora $(\dot{\mathrm{X}}=23,91 \mu 1)$, mientras que Columnea anisophylla registró el volumen de néctar más bajo ( $\dot{X}=6,58 \mu 1)$, (Figura 4).

El alto volumen de néctar producido por Guzmania multiflora y Cavendishia adenophora explican el mayor número de visitas de colibríes. Así, la alta producción de néctar de estas plantas puede ser una estrategia para que los polinizadores visiten un mayor número de sus flores, lo que concuerda con Murcia [14].

\section{CONCLUSIONES}

Se concluye que las cuatro especies de plantas estudiadas en la Finca Zíngara dependen de la visita obligada de los colibríes para la fecundación exitosa de sus flores.

Los colibríes son polinizadores efectivos de Guzmania multiflora y Cavendishia adenophora. Estas dos especies de plantas son autógamas y alógamas, producen alto volumen de néctar, tienen un alto número de interacciones con colibríes, quienes depositan principalmente polen legítimo en las flores que visitan. Las especies de Gesneriaceae, Columnea ferruginea y Columnea anisophylla, son autoincompatibles, con pocos individuos en floración, tuvieron una interacción con colibríes muy baja y exclusiva con colibríes ermitaños, lo que las hace especies especialistas. Estas características de Columnea spp. sugieren cierto grado de vulnerabilidad para sus poblaciones en la Finca Zíngara.

Se evidencia en este estudio, que para los colibríes fue más importante visitar flores de las especies que producen mayor cantidad de néctar, que flores de especies con mayor concentración de sacarosa.

\section{AGRADECIMIENTOS}

Las autoras agradecen a la Universidad del Valle por apoyar el desarrollo de esta investigación con el préstamo de los equipos y permitir el uso de las facilidades de la colección del Herbario CUVC, el Laboratorio de Semillas y el Laboratorio de Imágenes, del Departamento de Biología. Además, las autoras agradecen a Jorge Giraldo Gensini, por prestar el área de estudio y las facilidades de alojamiento en la Finca Zíngara.

\section{REFERENCIAS}

[1] P. Feinsinger, "Interacciones entre plantas y colibríes en selvas tropicales", Boletín de la Academia Nacional de Ciencias, Córdoba, Argentina, Tomo 59, entregas 1a $-2^{\mathrm{a}}, 1990$.

[2] L. Van der Pijl, "Ecological aspects of flower evolution. II. Zoophilous flower classes", Evolution, Vol. 15, pp. 44-59, 1961.

[3] P. K. Maruyama, G. M. Oliveira, C. Ferreira, B. Dalsgaard AND P. E. Oliveira, "Polli- 
nation syndromes ignored: importance of non-ornithophilous flowers to Neotropical savanna hummingbirds", Naturwissenschaften, Vol. 100, no. 11, pp. 1061-1068, 2013.

[4] C. Murcia, "Coevolución de los colibríes y las flores", en Joyas aladas de Colombia, L. Mazariegos (ed), Cali, Colombia: Imprelibros, pp. 120-130, 2000.

[5] L. Galetto AND G. Bernardello. "Nectar sugar composition in angiosperms from Chaco and Patagonia (Argentina): an animal visitor's matter?", Plant Syst. Evol., Vol. 238, pp. 69-86, 2003. https://doi.org/10.1007/ s00606-002-0269-y

[6] C. A. Kearns AND D. W. Inouye, Techniques for pollination biologists, Michigan: University Press of Colorado, 1993.

[7] W. A. Judd, C. S. Campbell, E. A. Kellog, P. F. Stevens AND M. J. Donoghue, Plant Systematics: a phylogenetic approach, 3ed., Massachusetts: Sinauer Associates, Inc, 2008.

[8] R. Paniagua, M. Nistral, P. Sesma, M. ÁlvaresUría, B. Fraile, R. Anadón Y F. Sáez, Citología e histología vegetal y animal, Madrid, España: Mc Graw-Hill Interamericana, 2002.

[9] A. H. D. Brown, "Genetic characterization of plant mating systems", in Plant populations genetics, breeding and genetic resources, A. H. D. Brown, A. Clegg, A. L. Kahler AND B. S. Weir (eds.), Massachusetts: Sinauer Associates, Vol. 225, no. 228-230, pp. 217-218, 1990.

[10] N. Myers, R. A. Mittermeier, C. G. Mittermeier, G. A. B. da Fonseca AND J. Kent, "Biodiversity hotspots for conservation priorities", Nature, Vol. 403, pp. 853-58, 2000.

[11] P. Bubb, I. May, L. Miles AND J. Sayer, Cloud Forest Agenda. UNEP-WCMC, Cambridge, UK, pp. 32, 2004.

[12] C. Eardley, D. Roth, J. Clarke, J. Buchmann AND B. Gemmill, Pollinators and Pollination: A resource book for policy and practi$c e$, African Pollination initiative \& United States Department of State, 2006.

[13] P. Feinsinger AND W. H Busby, "Pollen carryover: experimental comparisons between morphs of Palicourea lasiorrachis (Rubiaceae), a distylous, bird - pollinated, tropical treelet", Oecología, Vol. 73, no. 2, pp. 231-235, 1987.

[14] C. Murcia, "Ecología de la polinización", en Ecología y conservación de bosques neotropicales, M. R. Guariguata Y G. H. Kattan (eds.), Costa Rica, pp. 493-530. 2002.

[15] P. Feinsinger AND R. Colwell, "Community organization among neotropical nectarfeeding birds", American Zoologist, Vol. 18, pp.779-795, 1978.

[16] Y. B. Linhart, W. H. Busby, J. H. Beach, AND P. Feinsinger, "Forager behavior, pollen dispersal, and inbreeding in two species of hummingbird-pollinated plants", Evolution, Vol. 41, no. 3, pp. 679-682, 1987.

[17] P. Feinsinger, J. A. Wolfe, AND L. A. Swarm, "Island ecology: reduced hummingbird diversity and the pollination biology of plants, Trinidad and Tobago, West Indies", Ecology, Vol. 63, no. 2, pp. 494-506, 1982.

[18] A. Gutiérrez Y S. Rojas, "Dinámica anual de la interacción colibrí-flor en ecosistemas altoandinos del volcán Galeras, sur de Colombia”, Trabajo de Grado, Departamento de Biología, Universidad Nacional de Colombia, Bogotá.

[19] C. I. Rodríguez-Flores, Y F. G. Stiles, "Análisis ecomorfológico de una comunidad de colibríes ermitaños (Trochilidae, Phaethorninae) y sus flores en la amazonía colombiana", Ornitología Neotropical, Vol. 3, pp. 7-27, 2005.

[20] L. Rosero-Lasprilla Y M. Sazima, "Interacciones planta-colibrí en tres comunidades vegetales de la parte suroriental del Parque Nacional Natural Chiribiquete, Colombia", Ornitología Neotropical, Vol 15, pp. 183-190, 2004.

[21] M. Amaya-Márquez, F. G. Stiles Y O. Rangel-Gil, "Interacción planta-colibrí en Amacayacu (Amazonas, Colombia): una perspectiva palinológica", Caldasia, Vol. 23, no. 1, pp. 301-322, 2001.

[22] N. M. Waser AND V. Price, "Pollination efficiency and effectiveness of bumble bees and hummingbirds visiting Delphinium 
nelsonii", Collectana Botanica, Vol. 19, pp. 9-20, 1990.

[23] J. L. Stone, "Components of pollination efectiveness in Psychotria suerrensis, a tropical distylous shrub", Oecologia, Vol. 107, pp. 504- 512, 1996.

[24] M. A. Rocca AND M. Sazima, "Quantity versus quality: identifying the most effective pollinators of the hummingbird-pollinated Vriesea rodigasiana (Bromeliaceae)", Plant systematics and evolution, Vol. 299, no. 1 , pp. 97-105, 2013.

[25] C. Ferreira, P. K. Maruyama AND P. E. Oliveira, "Convergence beyond flower morphology? Reproductive biology of hummingbirdpollinated plants in the brazilian Cerrado", Plant Biology, Vol. 18, no. 2, pp. 316-324, 2015.

[26] F. Ayerbe-Quiñones, Colibries de Colombia. Serie: Avifauna colombiana, Wildlife Conservation Society, $352 \mathrm{p}$.

[27] K. R. Shivanna AND R. Tandon, Reproductive ecology of flowering plants: A manual, New Delhi, India: Springer, 2014.

[28] O. E. Murillo, "Efecto de la variación intraindividual en néctar sobre el comportamiento de alimentación de los colibríes y sobre el éxito reproductivo potencial de Palicourea lehmannii (Rubiaceae)". Tesis de Maestría, Postgrado en Ciencias - Biología, Universidad del Valle.

[29] G. Erdtman, Handbook of palynology: morphology, taxonomy, ecology.

[30] O. H. Marín-Gómez AND M. AmayaMárquez, "Diversidad, densidad poblacional y distribución espacial de Columnea (Gesneriaceae) en la Reserva Natural Río Ñambí, Nariño, Colombia", Revista de la Academia Colombiana de Ciencias Exactas, Físicas y Naturales, Vol. 39, no. 151, pp. 218-227, 2015. https://dx.doi.org/10.18257/ raccefyn. 145

[31] J. D. Ackerman, "Coping with the epiphytic existence: pollination strategies", Selbyana. Vol. 9, pp. 52-60, 1986.
[32] S. Bush and J. Beach, "Breeding systems of epiphytes in a tropical montane wet forest", Selbyana, Vol. 16, pp. 155- 158, 1995.

[33] J. L. Luteyn, "Diversity, Adaptation, and Endemism in Neotropical Ericaceae: Biogeographical Patterns in Vaccinieae". The Botanical Review, Vol. 68, no. 1, pp. 55-87, 2002.

[34] K. S. Bawa, "Breeding Systems of Tree Species of a Lowland Tropical Community", Evolution, Vol. 28, no. 1, pp. 85-92, 1974.

[35] N. M. Waser, "Interespecific pollen transfer and competition between co-occurring plant species", Oecología, Vol. 36, no. 2, pp. 223236, 1978.

[36] C. Violle, M. L. Navas, D. Vile, E. Kazakou, C. Fortunel, I. Hummel AND E. Garnier, "Let the concept of trait be functional", Oikos, Vol. 116, no. 5, pp. 882-892, 2007.

[37] C. Machado and J. Semir, "Fenologia da floração e biologia floral de bromeliáceas ornitófilas de uma área da Mata Atlántica do Sudeste brasileiro". Revista Brasilera de Botanica, Vol. 29, no. 1, pp. 163-174, 2006.

[38] P. Feinsinger, "Variable nectar secretion in a Heliconia species pollinated by hermit hummingbirds", Biotropica, Vol. 15, pp. 48-52, 1983.

[39] A. C. Araújo, E. A. Fischer and M. Sazima, "Floração seqüencial e polinização de três espécies de Vriesea (Bromeliaceae) na região da Juréia, sudeste do Brasill, Revista Brasileira de Botânica, Vol. 17, no. 2, pp. 113-118, 1994.

[40] E. A. Fischer and A. C. Araujo, "Spatial organization of a bromeliad community in the Atlantic Rainforest, South-Eastern Brazil", Journal of Tropical Ecology, Vol. 11, pp. 559-567, 1995.

[41] J. A. Carranza-Quiceno and J. V. EstévezVarón, "Ecología de la polinización de Bromeliaceae en el dosel de los bosques neotropicales de montaña", Boletín Científico Centro de Museos Museo de Historia Natural, Vol. 12, no 1, pp. 38-47, 2008. 
Angie Lisseth Martinez-Meneses - Alba Marina Torres-González

Tabla 1. Porcentaje de fructificación en los tratamientos de polinización de Cavendishia adenophora, Guzmania multiflora, Columnea ferruginea y Columnea anisophylla en la Finca Zíngara, Cali, Colombia

\begin{tabular}{lcccc}
\hline Tratamiento & Guzmania multiflora & Cavendishia adenophora & Columnea ferruginea & Columnea anisophylla \\
\hline Polinización cruzada natural & 80 & 60 & 50 & 50 \\
Polinización cruzada obligada & 60 & 70 & 0 & 50 \\
Autopolinización natural & 0 & 0 & 0 & 0 \\
Autopolinización obligada & 60 & 80 & 0 & 0 \\
\hline
\end{tabular}
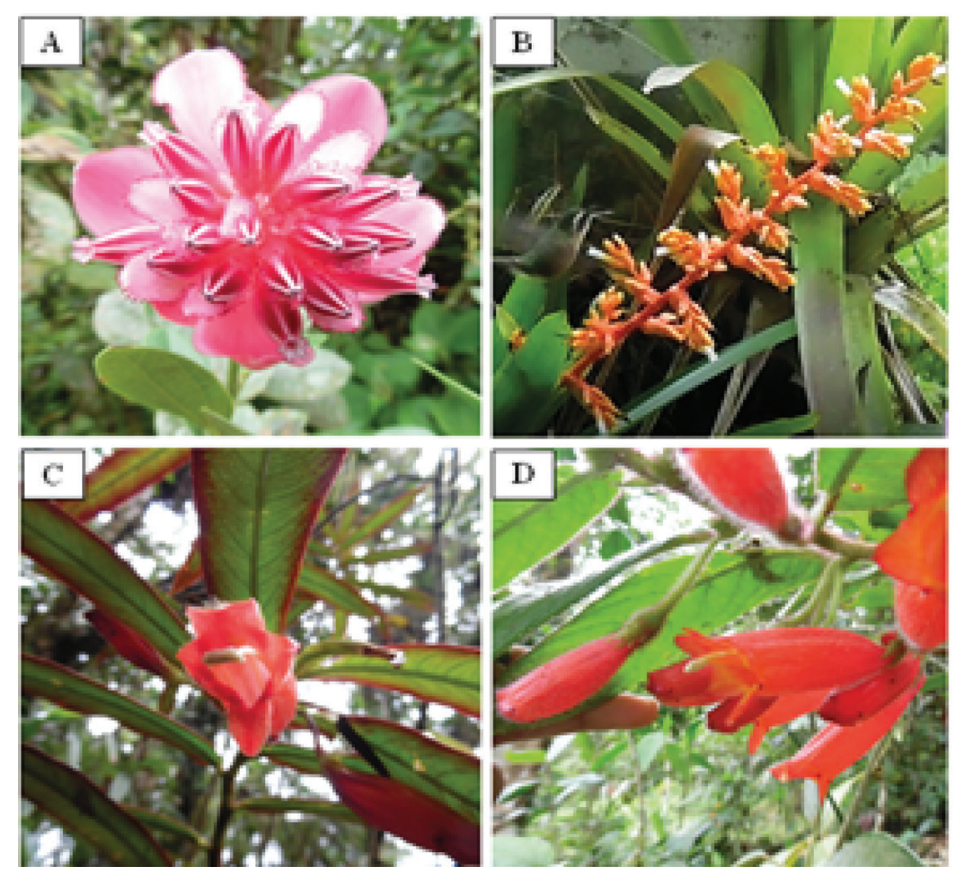

Figura 1. Especies de plantas estudiadas en la finca Zingara, Cali, Colombia. A. Cavendishia adenophora, B. Guzmania multiflora, C. Columnea ferruginea y D. Columnea anysophylla. 


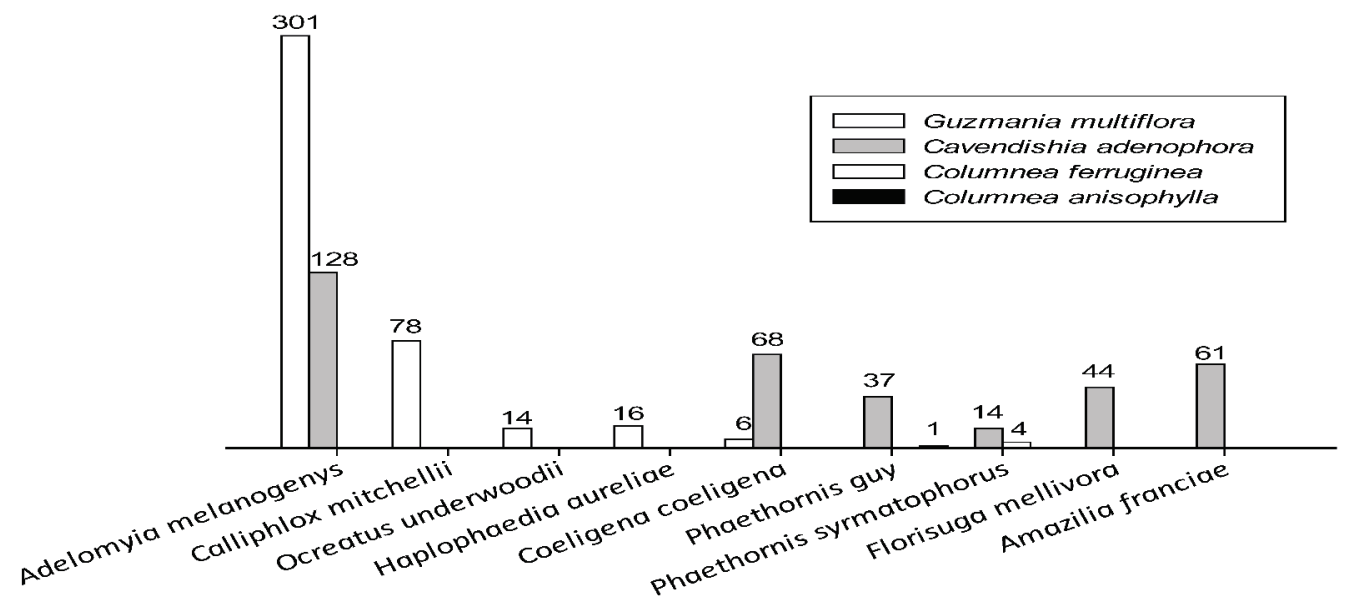

Figura 2. Número de visitas de colibríes registradas a las flores de Guzmania multiflora (barra blanca), Cavendishia adenophora (barra gris), Columnea ferruginea (barra punteada) y Columnea anisophylla (barra negra) en la Finca Zíngara, Cali, Colombia. Tiempo de observación: 174 h.

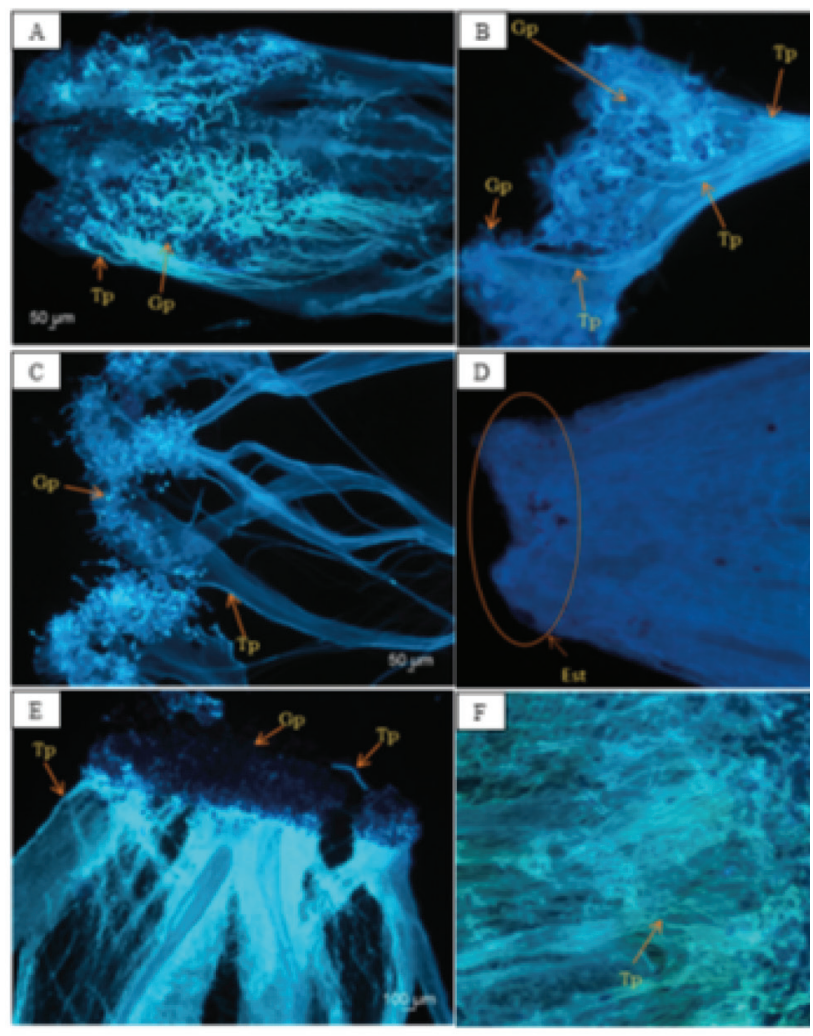

Figura 3. Crecimiento de tubos polínicos vistos en el microscopio de epifluorescencia, en las plantas estudiadas en la finca Zíngara, Cali, Colombia. A. Autopolinización obligada en Guzmania multiflora, se observa gran densidad de polen y tubos polínicos en el estigma. B. Autopolinización natural en Guzmania multiflora, los tubos polínicos llegan hasta el estilo. C. Polinización cruzada obligada en Guzmania multiflora, los tubos polínicos llegan hasta el ovario. D. Autopolinización natural en Cavendishia adenophora, no se observa ningún grano de polen adherido al estigma. E. Polinización cruzada obligada en Columnea anisophylla, se observa gran densidad de polen sin germinar y solo dos tubos polínicos. F. Polinización cruzada obligada en Columnea ferruginea, muchos tubos polínicos que crecieron hasta el ovario. Gp: grano de polen, Tp: tubo polínico en crecimiento, Est: estigma. Fotos: Juan Felipe Ortega. 
Angie Lisseth Martinez-Meneses - Alba Marina Torres-González

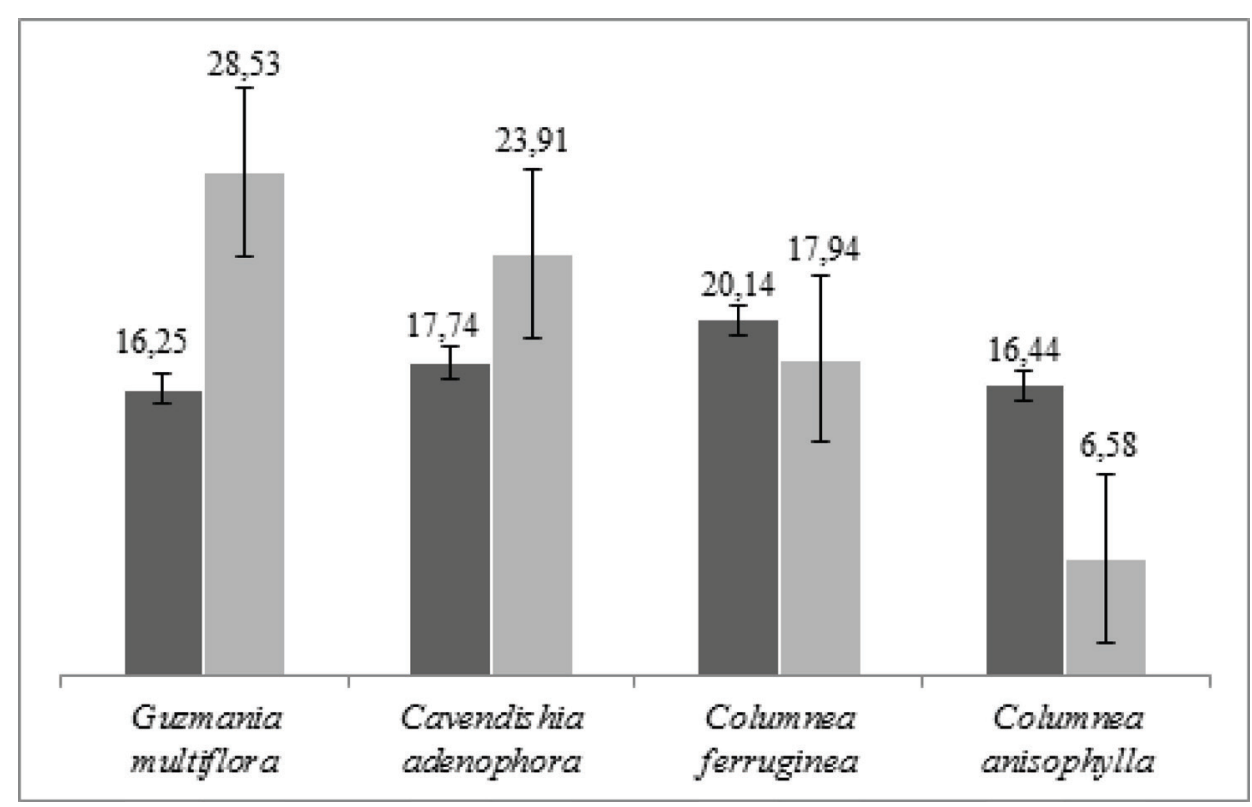

Figura 4. Concentración de sacarosa en el néctar $\left({ }^{\circ}\right.$ Brix, barra gris oscuro) y volumen de néctar acumulado durante el día ( $\mu \mathrm{L}$, barra gris claro) en las flores de Cavendishia adenophora, Guzmania multiflora, Columnea ferruginea y Columnea anisophylla en la Finca Zíngara, Cali, Colombia. 
